\title{
SIMPLE SVM BASED WHOLE-GENOME SEGMENTATION
}

\author{
JUSTIN BEDO, GEOFF MACINTYRE, IZHAK HAVIV, AND ADAM KOWALCZYK
}

\begin{abstract}
We present a support vector machine (SVM) based framework for DNA segmentation into binary classes. Two applications are explored: transcription start site prediction and transcription factor binding prediction. Experiments demonstrate our approach has significantly better performance than other methods on both tasks.
\end{abstract}

\section{INTRODUCTION}

Recently, there has been a substantial increase in annotations for the human genome. This is in part due to the next-generation sequencing revolution that has substantially increased the amount of data available. Now, high-quality annotations of the genome are available in a quantity that was previously unheard of and the main challenge is to mine this data for useful information.

Classical approaches such as motif detection and position weight matrices (PWM) have modelled biological processes through the collection of short sequences and generation of motifs using alignment. These motif models are then used to predict activity on new genomic.

This paper presents a simple SVM based framework for segmenting and labelling the entire genome into binary classes. We explore two applications, one in transcription start site (TSS) prediction and the other in transcription factor binding site (TFBS) prediction. Experiments show our simple approach outperforms another more complex SVM based predictor for TSS prediction, and also outperforms the commonly used PWMs for TFBS.

\section{Method}

Let $\vec{s} \in\{a, g, t, c\}^{n}$ be the sequence under analysis and let $\vec{l} \in\{-1,1\}^{n}$ be a corresponding label vector. We shall assume the minority class is given the positive label 1 . Consider the consecutive segments $\vec{x}_{i} \in\{a, g, t, c\}^{\omega}$ and $\vec{y}_{i} \in\{-1,1\}^{\omega}$ of fixed length $\omega$. The problem is to label each segment $x_{i}$ into one of two classes derived from the nucleotide labels $\vec{l}$. In this paper we shall consider labelling schemes of the form $\bar{y}_{i} \triangleq\left[\sum_{j=1}^{\omega} y_{i j}>\tau\right]$.

There are three simple schemes arising from such labelling. The first is to take $\tau=0$. This corresponds to labelling any segment containing a majority of positive nucleotides as a positive segment, with the remaining segments forming the negative samples. This scheme would be suitable for nucleotide labels $\vec{l}$ where the positive labels form contiguous blocks of large length greater than the window size $\omega$.

The second scheme is to take $\tau=-\omega$. This corresponds to labelling all segments containing at least one positive base as a positive segment. This scheme can be used with both point and contiguous block labels.

The final scheme is to take $\tau=\omega$ which corresponds to labelling only windows completely contained within the positive segment as positive. This form of labelling may be useful for applications with large positive regions or small window sizes $\omega$.

Features for learning were generated from the sequences $\vec{x}_{i}$ using $k$-mers, which we define as the frequency count of all sub-sequences of $k$ length where $k \ll \omega$. We use $\phi:\{a, g, t, c\}^{m} \rightarrow \mathbb{N}^{4^{k}}$ to denote the map between sequences and the $k$-mer feature space. 
We used a linear least-squares support vector machine (SVM) [Schölkopf and Smola, 2002] to classify each segment $\vec{x}_{i}$ with binary labels $\bar{y}_{i}$. The linear prediction function for the $i^{\text {th }} \frac{\text { segment }}{\text { segen }}$ is thus 1 .

$$
f\left(\vec{x}_{i}\right) \triangleq\left\langle\bar{\phi}\left(\vec{x}_{i}\right), \vec{\beta}\right\rangle
$$

where $\vec{\beta} \in \mathbb{R}^{4^{k}}$. The weights $\vec{\beta}$ are found by minimising the objective

$$
\arg \max _{\vec{\beta}} \Xi(\vec{\beta}) \triangleq \frac{1}{2} \sum_{i} \max \left(1-\bar{y}_{i}\left\langle\bar{\phi}\left(\vec{x}_{i}\right), \vec{\beta}\right\rangle, 0\right)^{2}+\frac{1}{2} \lambda\|\vec{\beta}\|^{2},
$$

where $\lambda$ is the regularisation hyperparameter. If we let $X$ denote a matrix where the $i^{\text {th }}$ row is the sample $\bar{\phi}\left(\vec{x}_{i}\right)$ in feature space and $Y$ denote the vector $\left[\bar{y}_{i}\right]$, then we can write $\Xi$ in matrix form as

$$
\Xi(\vec{\beta}) \triangleq \frac{1}{2}(X \vec{\beta}-Y)^{T} I(X \vec{\beta}-y)+\frac{1}{2} \lambda\|\vec{\beta}\|^{2},
$$

where $I$ is a diagonal matrix with entries $I_{i i} \triangleq 1-\bar{y}_{i}\left\langle\bar{\phi}\left(\vec{x}_{i}\right), \vec{\beta}\right\rangle$.

Minimisation of $\Xi$ can be done for small $k$ easily in the primal domain. This comprises of iterating

$$
\vec{\beta}_{t+1} \leftarrow\left(X^{T} I_{t} X \vec{\beta}_{t}+\Lambda\right)^{-1} X^{T} I_{t} Y
$$

where $\Lambda$ is a diagonal matrix with entries $\Lambda_{i i} \triangleq \lambda$. This is a variant of the well-known ridgeregression solution [Hastie et al. 2001] with the additional $I$ matrix. This is effectively a descent along the subgradient of $\Xi$.

For large $k, \Xi$ can still be minimised using a large-scale SVM learning algorithm such as the Pegasos algorithm Shalev-Shwartz et al. 2007. All experiments in this paper were conducted using the primal solver described above.

To evaluate model performance, two metrics were used. The first is the receiver operating characteristic (ROC) and the area under the ROC (AUC) Hanley and McNeil, 1982. The ROC is defined as the plot of the true positive rate (TPR) $T P / \overline{(T P+F N)}$ vs the false positive rate (FPR) $F P /(F P+T N)$ as the decision threshold is varied ${ }^{2}$. The AUC is the area under this curve and has been shown to be equivalent to the probability of correctly ordering class pairs Hanley and McNeil, 1982. I.e., the AUC of an hypothesis $f$ can be calculated by

$$
\operatorname{aroc}(f)=P_{(\vec{x}, \bar{y}),\left(\vec{x}^{\prime}, \bar{y}^{\prime}\right)}\left[f(\vec{x})>f\left(\vec{x}^{\prime}\right) \mid \bar{y}=1 \& \bar{y}^{\prime}=-1\right] .
$$

The second metric we use is the precision-recall curve (PRC). The precision is defined as the true discovery rate $(T P /(T P+F P))$ and the recall is equivalent to the TPR. Similarly to the ROC, we take the area under the PRC (APRC) as a general measure of the performance across all thresholds.

To reduce the number of features used in the model, we combined the SVM with recursive feature elimination (RFESVM) Guyon et al., 2002. The RFESVM eliminates features by obtaining the SVM weights $\beta$ using the above procedure followed by discarding the feature(s) with the smallest magnitude $\left|\beta_{i}\right|$. This process is then repeated recursively until a model of the desired size is obtained. To accelerate the process, $10 \%$ of the worst features were discarded when the model size was above 100 features and individually discarded when below. To optimise the model size and regularisation parameter $\lambda$, 3-fold cross-validation Hastie et al., 2001] was used with a grid search for $\lambda$, and the model with greatest average APRC chosen.

\footnotetext{
${ }^{1}$ The typical bias term has been absorbed into the weights $\vec{\beta}$ for simplicity. This is a standard trick that involves adding a constant feature of value 1 to all samples.

${ }^{2} T P, F P, T N$ and $F N$ are the number of true positives, false positives, true negatives and false negatives respectively.
} 


\section{Applications}

The simple SVM approach was evaluated on two different classification problems: transcription start site (TSS) and transcription factor binding (TFB) prediction. In all experiments, we trained using only samples from chromosome 22 and then predicted on the whole-genome.

3.1. Transcription start site prediction. The location of genes which encode proteins is important information. The location of these in-silico would be of benefit to the research community. Supervised learning has been applied to the TSS detection in the past, most notably by Sonnenburg et al. 2006a]. Sonnenburg et al. 2006a also approached the problem using SVMs, but their framework is far more complicated. In our case, we use a very simple feature space of $k$-mers calculated directly over a region of the genome. Their approach uses multiple kernels, of which a $k$-mer kernel is applied both upstream and downstream of the TSS along with a weighted degree kernel with shifts Sonnenburg et al., 2006b], which is quite complex and difficult to compute. Indeed, Sonnenburg et al. [2006a] state that approximately 350 hours are required to scan the whole genome.

In comparison, the approach here is vastly simpler and works directly in a linear feature space with dimension considerably smaller than kernel space. In particular, we well see that extremely short $k$-mers provide good performance and have sufficiently low dimensionality to be able to scan the whole genome in minutes not hours.

The first experiment was to replicate experimental procedure $1 \mathrm{~B}$ of Abeel et al. 2009. I.e., we took the RefSeq gene databas $\epsilon^{3}$ as our labels, used a window size of $\omega=500$ and labelled each window overlapping a positive RefSeq label as positive $(\tau=-\omega$ as described in the methods section). Furthermore, only negative segments intersecting exonic regions were used.

Figure 1 shows the results of the experiment. Abeel et al. 2009. compared against many predictions methods, of which the best was ARTS [Sonnenburg et al., 2006a] with an APRC of $36 \%$, which was $9 \%$ better than the 2 nd best performer. Our simple SVM method obtained the PRC curve shown in Subfigure 1a with an APRC of $48 \%$. This is vastly superior performance to that of ARTS.

We now turn to whole-genome analysis using the RefSeq database with results shown in Figure 2. As expected, the overall performance is lower than with the exon-restricted negative samples. One may hypothesise two reasons for this; firstly, not all of the negatively labelled samples will be true negatives, and secondly the DNA contains more variety than the exon regions only.

One curious point of note is the sharp decline in precision that can be observed as recall $\rightarrow$ 0 in Subfigure 2a. This can only be caused by the most confidently predicted samples being incorrect. One hypothesis is that these are in fact true positives that have been incorrectly labelled as negative. Supportive of this may be that this decline is not observable when using exon-restricted nulls in Subfigure 1a. We have not yet explored this hypothesis further.

3.2. Transcription factor binding site prediction. The second application we explored was transcription factor binding site prediction. In this case, the sequence labels were derived using ChIP-Seq data from the ENCODE project. For comparison, position frequency matrices obtained from the TRANSFAC database were used with the position weight matrix (PWM) method of Wasserman and Sandelin 2004.

The first transcription factor we evaluated was c-Myc. ENCODE TFBS data from the Yale on the K562 cell-line was used 4 Figure 3 shows the results of training on chromosome 22 and testing on the whole-genome. The blue line is our RFESVM approach, and the remaining lines are

\footnotetext{
${ }^{3}$ http://refgene.com/

${ }^{4}$ http://genome.ucsc.edu/ENCODE
} 
different PWMs obtained from TRANSFAC. It is clear from Subfigure 3a that the performance of the SVM approach is vastly superior to the PWMs.

The second transcription factor is C-Fos with results shown in Figure 4. Again, the Yale ENCODE data on the K562 cell-line was used. The general performance is much lower on this transcription factor indicating it is a more difficult target than c-Myc. Nevertheless, the RFESVM approach still dominates and performs the best by a large margin.

\section{Conclusions}

We have presented a general framework for segmenting and classifying the whole-genome into binary classes. This framework was applied to TFBS and TSS prediction. In both cases, our SVM framework demonstrated significantly greater predictive performance than existing methods.

This substantial increase in performance suggests that supervised machine learning is a serious contender for genomic segmentation. For TFBS prediction, motifs are generally built by extracting small bound segments of DNA followed by alignment and determination of a PFM using tools such as MEME Bailey et al. 2006. These approaches are sensitive to the input sequences and must therefore be chosen very carefully Bailey et al., 2006. This explains the plethora of different PFMs available in databases such as TRANSFAC for the same transcription factor: using different bound sequences results in (potentially vastly) different PFMs.

We have demonstrated in our experiments that PWMs do not perform well for prediction of TFBS sites. In contrast, our method approaches the problem in a far simpler way and automatically extracts short "motifs" (the $k$-mer features). Though each individual $k$-mer is less powerful in modelling patterns than PFMs, by using a collection of them the SVM is able to model complex patterns, more-so than PFMs. Additionally, as large windows are used, the models have the ability to discover the necessary sequence conditions for binding; i.e., the SVM approach has the power to model co-binding factors and other phenomenon.

Finally, we remark that the next best performer for TSS prediction as demonstrated by Abeel et al. 2009] is the ARTS method [Sonnenburg et al., 2006a which again approaches the problem using supervised learning. This is further evidence that the supervised approach is powerful and well suited to sequence problems, especially with the rise of available genomic annotations. However, though our approaches are similar philosophically, we differ in that our SVM model is far simpler. We only use simple $k$-mer features with no other kernels and no assumptions nor incorporation of upstream and downstream features.

That such simple methods perform so well is encouraging. It provides us with baseline performance results at low computational cost and demonstrates Occam's razor in the context of learning. We should explore more powerful learning models able to capture more of the true biological processes, however we should not forget about finding simple models with good performance that are easily interpretable.

\section{Author CONTRIBUtions}

JB developed and implement the methodology, ran all experiments and wrote this manuscript.

AK suggested the transcription start site prediction application and prepared the RefSeq transcription start site data.

IH suggested the transcription factor binding site prediction application.

GM prepared the ENCODE transcription factor data and the PFMs from TRANSFAC.

\section{ACKNOWLEDGEMENTS}

JB, AK and GM acknowledge the support of NICTA in conducting this research. NICTA is funded by the Australian Government's Department of Communications, Information Technology 
and the Arts, the Australian Research Council through Backing Australia's Ability, and the ICT Centre of Excellence programs.

\section{REFERENCES}

Bernhard Schölkopf and Alexander J. Smola. Learning with Kernels. MIT Press, 2002.

Trevor Hastie, Robert Tibshirani, and Jerome Friedman. The Elements of Statistical Learning. Springer, 2001.

S Shalev-Shwartz, Y Singer, and N Srebro. Pegasos: Primal estimated sub-gradient solver for svm. Proceedings of the 24th international conference on Machine Learning, pages 807-814, 2007.

$\mathrm{J}$ A Hanley and B J McNeil. The meaning and use of the area under a receiver operating characteristic (roc) curve. Radiology, 143(1):29-36, Apr 1982.

IM Guyon, J Weston, S Barnhill, and V Vapnik. Gene selection for cancer classification using support vector machines. Machine Learning, 46:389-422, Nov 2002.

S Sonnenburg, A Zien, and G Ratsch. Arts: accurate recognition of transcription starts in human. Bioinformatics, 22(14), 2006a.

S Sonnenburg, G Rätsch, C Schäfer, and B Schölkopf. Large scale multiple kernel learning. JMLR, 7:1531-1565, 2006b.

Thomas Abeel, Y Van de Peer, and Y Saeys. Toward a gold standard for promoter prediction evaluation. Bioinformatics, 25:i313-i320, Jan 2009.

Wyeth W Wasserman and Albin Sandelin. Applied bioinformatics for the identification of regulatory elements. Nature Review Genetics, 5(4):276-287, 2004.

T Bailey, N Williams, C Misleh, and W Li. Meme: discovering and analyzing dna and protein sequence motifs. Nucleic Acids Res, 34, Jan 2006.

NiCta Victoria Research Laboratory, Lvl 2 / Bldg 193, The University of Melbourne, ViC 3010 Australia

E-mail address: bedo@ieee.org

E-mail address: gmaci@csse.unimelb.edu.au

E-mail address: a.kowalczyk@ee.unimelb.edu.au

Baker IDi Heart and Diabetes Institute, PO Box 6492, St Kilda Road Central, ViC 8008, Australia

E-mail address: izhak.haviv@bakeridi.edu.au 


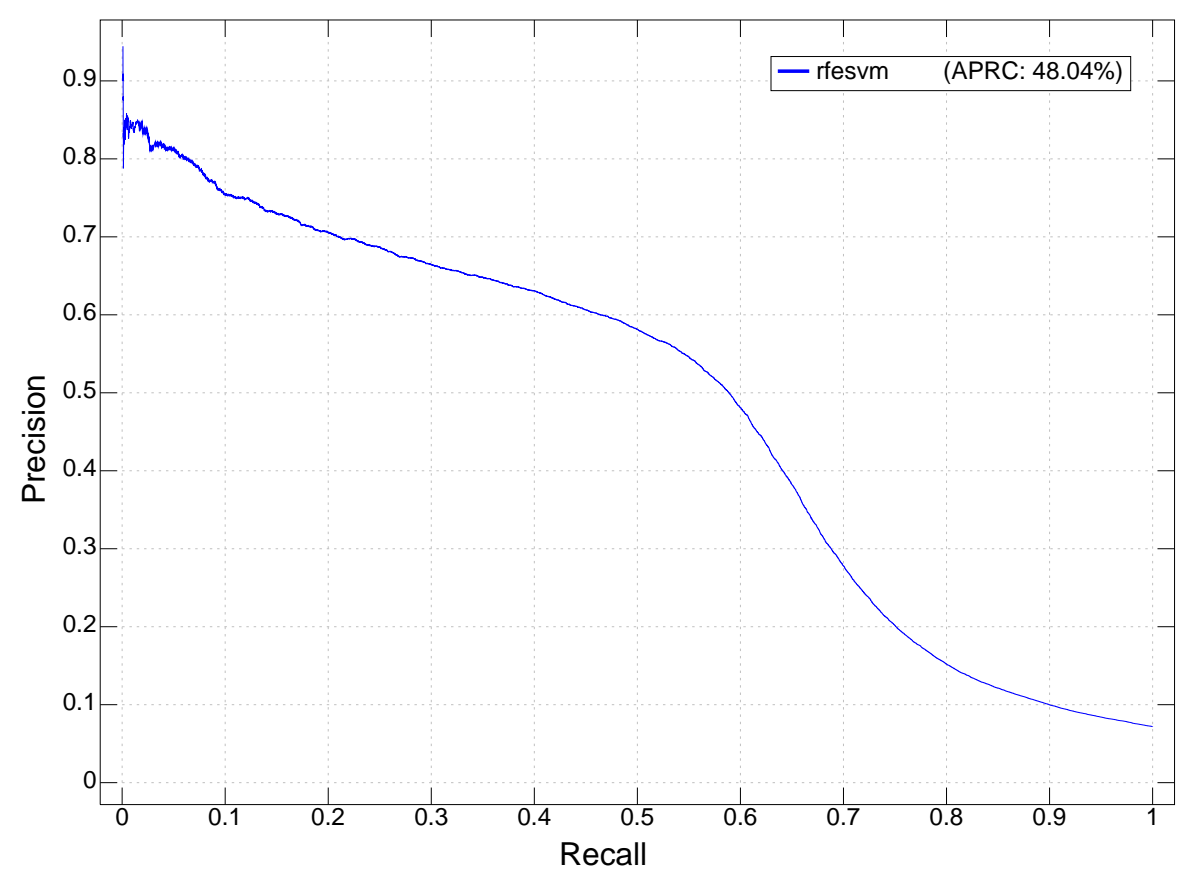

(a) PRC

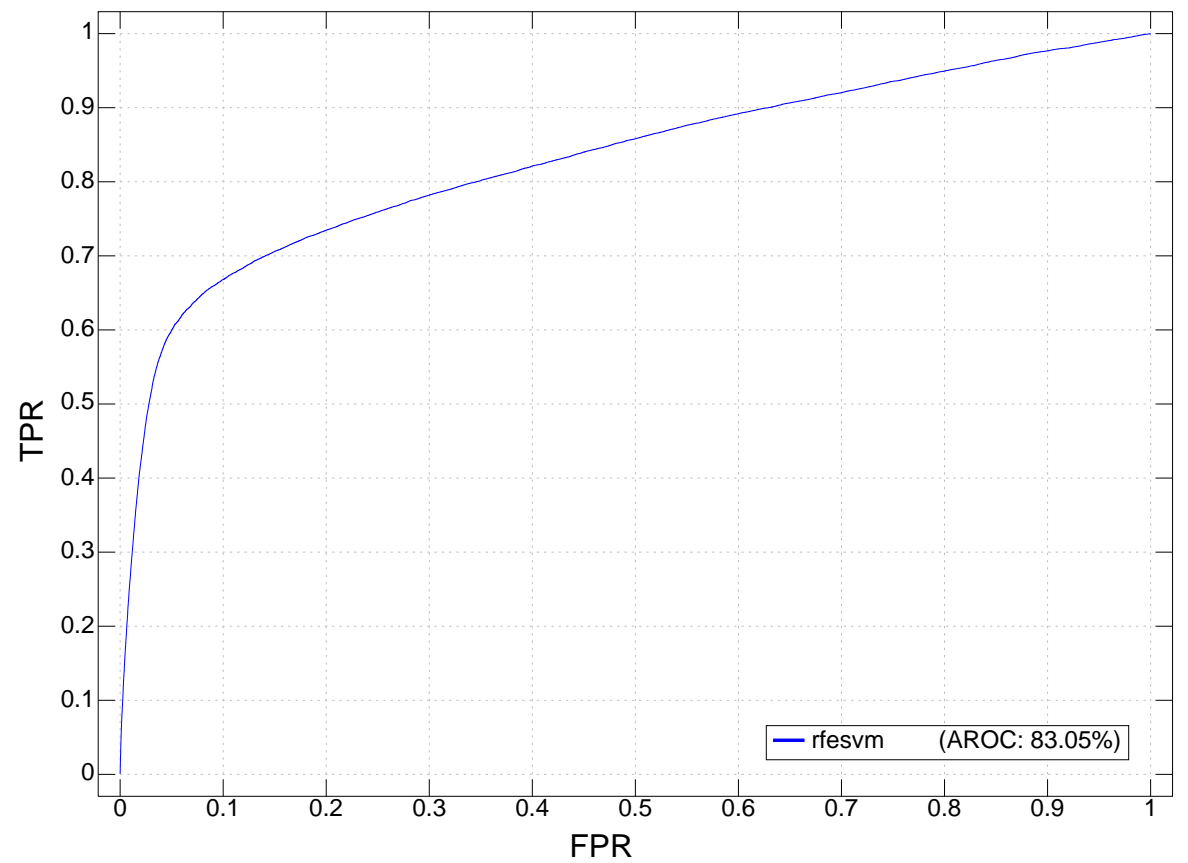

(b) ROC

Figure 1: Performance curves for the RefSeq TSS database using exon-restricted negative samples only 


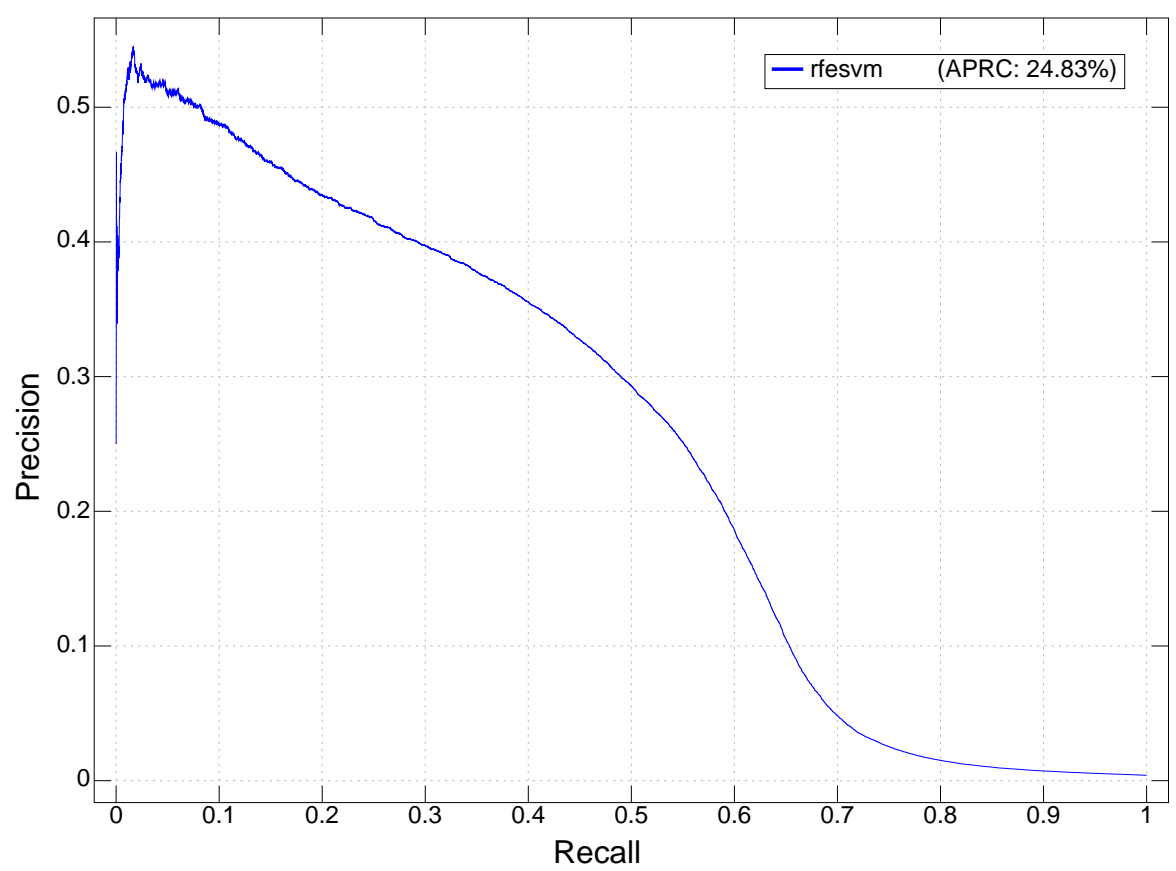

(a) PRC

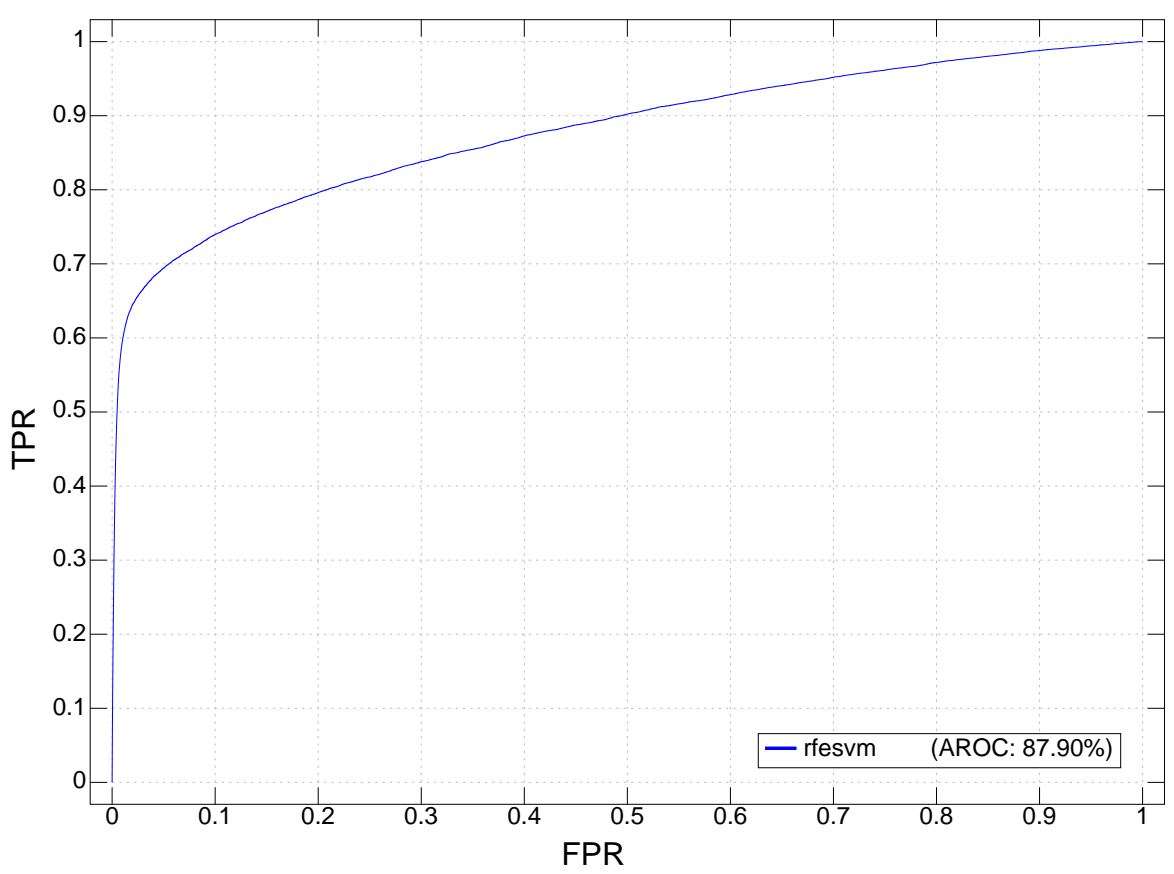

(b) ROC

Figure 2: Whole-genome performance curves for the RefSeq TSS database 


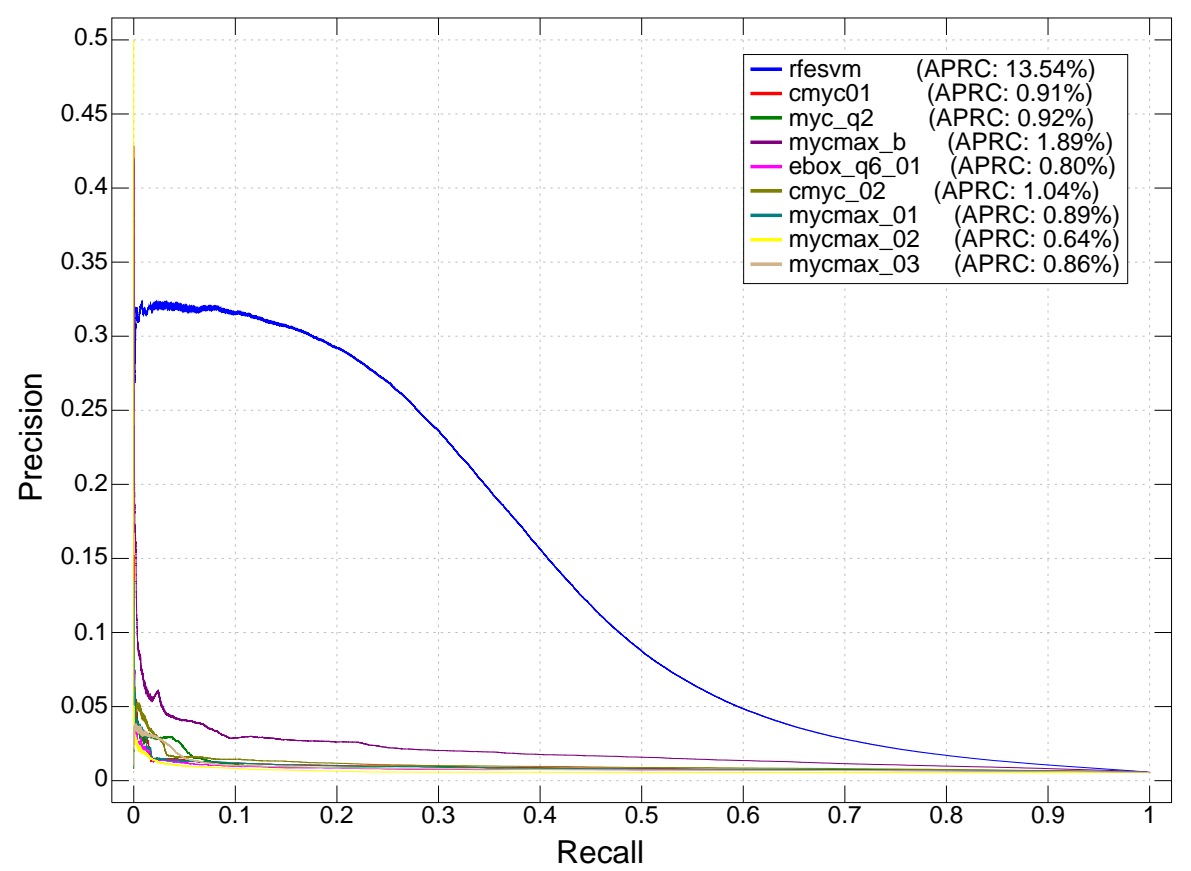

(a) PRC

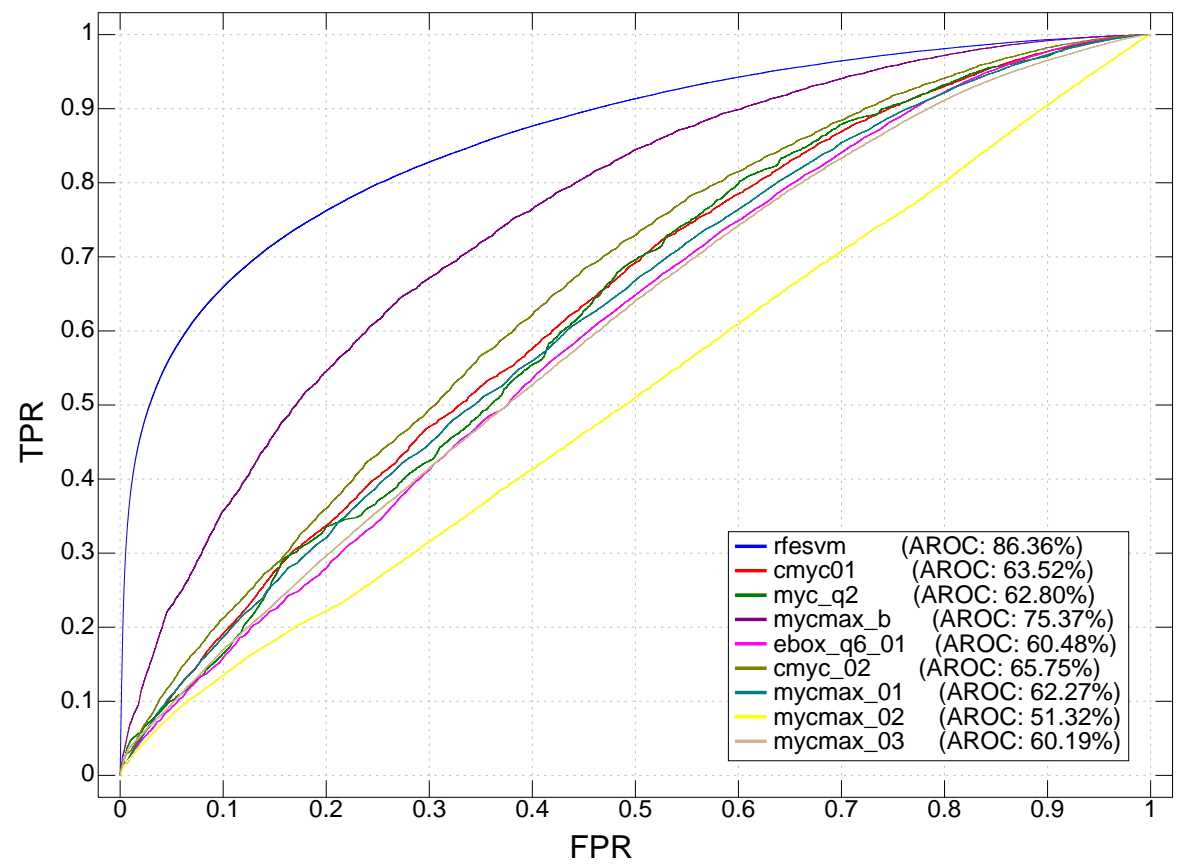

(b) ROC

Figure 3: Whole-genome PRC curve for c-Myc transcription factor. The blue plot is our RFESVM method, the remaining are various position weight matrices. 


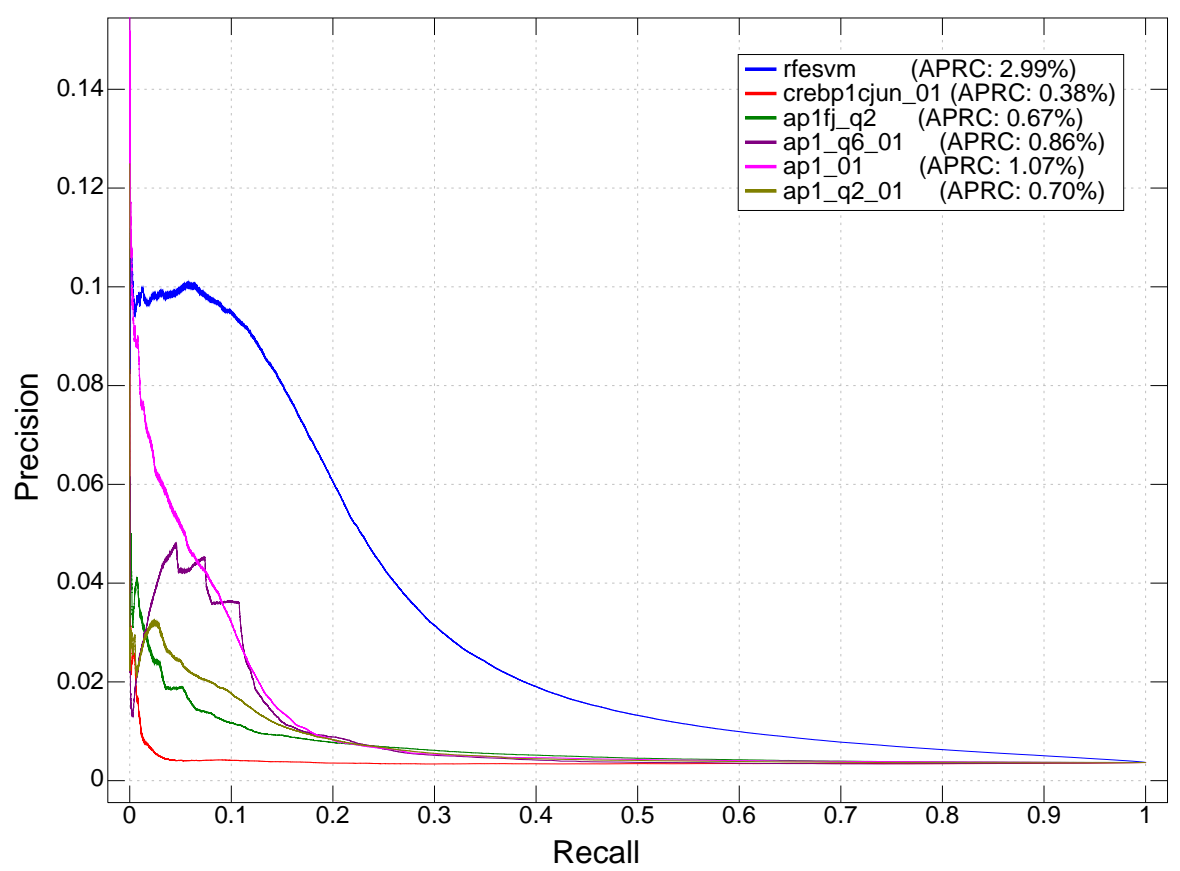

(a) PRC

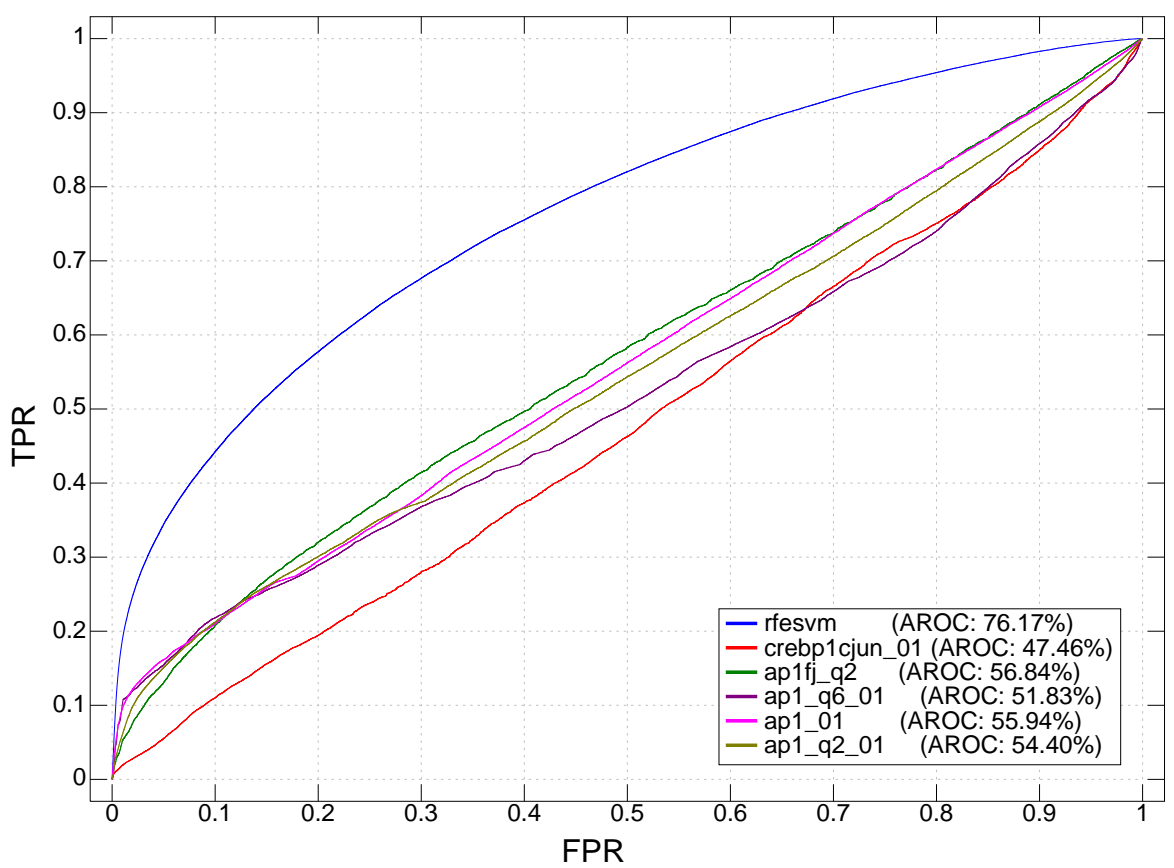

(b) ROC

Figure 4: Whole-genome PRC curve for C-Fos transcription factor. The blue plot is our RFESVM method, the remaining are various position weight matrices. 